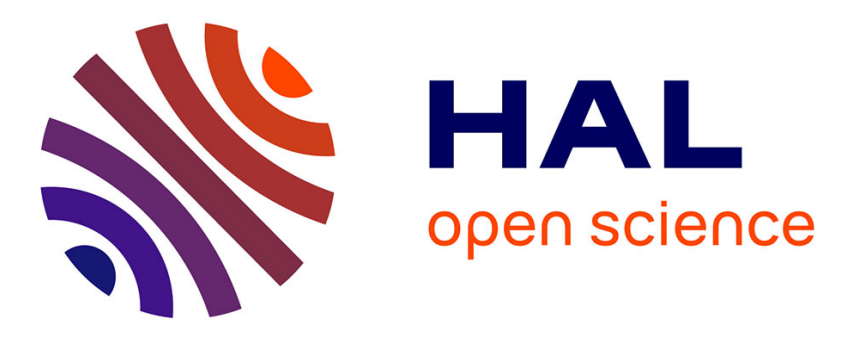

\title{
An Adaptable Framework to Deploy Complex Applications onto Multi-cloud Platforms
}

Linh Manh Pham, Alain Tchana, Didier Donsez, Vincent Zurczak, Pierre-Yves Gibello, Noel de Palma

\section{- To cite this version:}

Linh Manh Pham, Alain Tchana, Didier Donsez, Vincent Zurczak, Pierre-Yves Gibello, et al.. An Adaptable Framework to Deploy Complex Applications onto Multi-cloud Platforms. Computing \& Communication Technologies - Research, Innovation, and Vision for the Future (RIVF), 2015 IEEE RIVF International Conference on, Jan 2015, Can Tho, Vietnam. 10.1109/RIVF.2015.7049894 . hal01232615

\section{HAL Id: hal-01232615 https://hal.science/hal-01232615}

Submitted on 23 Nov 2015

HAL is a multi-disciplinary open access archive for the deposit and dissemination of scientific research documents, whether they are published or not. The documents may come from teaching and research institutions in France or abroad, or from public or private research centers.
L'archive ouverte pluridisciplinaire HAL, est destinée au dépôt et à la diffusion de documents scientifiques de niveau recherche, publiés ou non, émanant des établissements d'enseignement et de recherche français ou étrangers, des laboratoires publics ou privés. 


\title{
An Adaptable Framework to Deploy Complex Applications onto Multi-cloud Platforms
}

\author{
Linh Manh Pham ${ }^{1}$, Alain Tchana ${ }^{2}$, Didier Donsez ${ }^{1}$, Vincent Zurczak ${ }^{3}$, Pierre-Yves Gibello ${ }^{3}$, Noel de Palma ${ }^{1}$ \\ ${ }^{1}$ University of Joseph Fourier, Grenoble, France. E-mail: first.last@imag.fr \\ ${ }^{2}$ University of Toulouse, Toulouse, France. E-mail: first.last@enseeiht.fr \\ ${ }^{3}$ Linagora, Grenoble, France. E-mail: (vincent.zurczak, pygibello)@linagora.com
}

\begin{abstract}
Cloud computing is nowadays a popular technology for hosting IT services. However, deploying and reconfiguring complex applications involving multiple software components, which are distributed on many virtual machines running on single or multi-cloud platforms, is error-prone and time-consuming for human administrators. Existing deployment frameworks are most of the time either dedicated to a unique type of application (e.g. JEE applications) or address a single cloud platform (e.g. Amazon EC2). This paper presents a novel distributed application management framework for multi-cloud platforms. It provides a Domain Specific Language (DSL) which allows to describe applications and their execution environments (cloud platforms) in a hierarchical way in order to provide a fine-grained management. This framework implements an asynchronous and parallel deployment protocol which accelerates and make resilient the deployment process. A prototype has been developed to serve conducting intensive experiments with different type of applications (e.g. OSGi application and ubiquitous big data analytics for IoT) over disparate cloud models (e.g. private, hybrid, and multi-cloud), which validate the genericity of the framework. These experiments also demonstrate its efficiency comparing to existing frameworks such as Cloudify.
\end{abstract}

Keywords-Middleware/business logic, Software Engineering/Management

\section{INTRODUCTION}

For many decades now, we are seeing the continuous growth of the complexity of applications, due to the development of new technologies on the one hand, and the emergence of new needs on the other hand. An application does not address a single problem but several. This growth of their complexity implies the same phenomenon regarding their execution environment on organization of physical machines or devices. For instance, this has brought forward a change from centralized to distributed and heterogeneous place of execution. All of this makes human administration very difficult because they are errors prone, slow to respond (e.g. fault solving), and highly costly (e.g. wages). In the early 2000s, IBM [1] proposed to automate their administration throughout the use of what we called Autonomic Computing Systems (ACS for short). This practice consists in transferring human administration knowledge and behaviors to computing systems. This can be done in two ways, either by introducing autonomic behaviors into applications components at its implementation time (e.g. [9]) or by building a computing system (different to the application we want to administrate) which will make the application autonomous [4]. In this paper we consider the second way. As summarized by [1], administration tasks can be divided into the following categories: (un)installation and reconfiguration. The former includes the initialized provisioning of the execution environment, the installation of artifacts from repositories, the configuration of the application, its start-up as well as stopping and releasing allocated resources when needed. About reconfiguration tasks, they are performed at runtime in order to reconfigure the application when a particular situation is detected (e.g. fault). ACSs have proved their usefulness and now the major part of research in this topic focuses on reconfigurations tasks [2].

However, recent years have seen the development of a new technology called Cloud computing which is a challenging domain for existing ACSs, as it introduces an intermediate level of administration for virtual machines (VMs). Moreover, it sometimes requires the utilization of several clouds at once (hybrid and multi-cloud). To make matter worse, clouds API are not standardized, which results to non interoperable clouds. For example, running an enterprise financial/bank application within the Cloud generally requires two clouds: a private cloud (e.g. vSphere or OpenStack) located in the company to run business-critical part and a public cloud (e.g. EC2 or Azure) to run non-critical part. Note that in some situations, the latter part can move from one cloud to another for price and competitiveness reasons.

In this context, existing ACSs [3], [6] are inappropriate for several reasons. (1) Existing ACSs only consider one level of deployment/execution: an application runs within a physical machine, whereas in the subject of the Cloud, the application runs within a VM or a container, which in turn runs on a physical machine. (2) The target execution environment is not static in the context of the Cloud, an application does not stay within the same cloud during its overall lifetime. (3) Existing ACSs are built to administrate both application and execution environment while in the context of cloud, administration is ensured by two actors: the deployer administrates its application while the cloud provider administrates VMs and physical machines. Although generic ACSs [4] for grids and clusters of machines exist, their enhancement for clouds requires a high expertise for the deployer. Concerning cloud solutions, they [14], [15], [16] are either proprietary, devote to a specific application, or target a static cloud.

This paper addresses these problems by proposing a generic (administrate any kind of applications), extensible, multi-cloud (target several clouds at once), scalable, and fine-grained reconfigurable deployment framework. The key ideas behind the framework are the following: (1) It is the composition of a ACS 
light-weight kernel which implements basic administration mechanisms; (2) a set of reusable components which are easily improvable by any deployer; and (3) a hierarchical DSL (Domain Specific Language) for a fine-grained expression of applications and execution environments. To keep the thing simple, we focus on a subset of administration stacks: initial installation (including provisioning, configuration, start-up, software deployment, stopping and uninstallation), dynamic installation at runtime, and incrementally partial or full application installation. In summary, we make the following contributions in this paper: (1) introduce a generic, improvable, and scalable deployment framework for multi-cloud applications. The prototype of this system is open source ${ }^{1}$ and is actually in use by several enterprises [32]; (2) perform several experiments which validate all the properties of the proposed framework. We deploy a web application onto a hybrid cloud (a private VMware vSphere hosting center combined with Amazon EC2 [33] and Azure [34] clouds). We also deploy an application utilizing for Home Automation (OpenHAB [35]) on embedded board, EC2 and Azure clouds; (3) propose a hierarchical DSL which allows fine-grained administration.

The rest of the paper is organized as follows. Section II presents the related work. Section III presents architecture of our deployment system. The evaluation and results are presented in Section IV. Lastly, Section V concludes the paper.

\section{RELATED WORK}

A lot of research works [7] have been devoted to the automation of the administration of distributed applications in cluster or grid environments. Some of them [3], [6] have focused on the installation phase. [5] presents a survey of software installation until 2007. Since the introduction of the Cloud computing technology, most research in the domain of autonomic computing for grid systems have been refocused. This section presents what is done for Cloud platforms.

As for grid systems, a number of research in the context of Cloud computing are dedicated either to a single cloud platform or a single type of applications. [8], [12] present a set of deployment frameworks in order to provide scalable testing solutions based on the Cloud. [10] focuses on fault tolerance on machine, VM and software component at runtime. Like our work, it relies on a publish/subscribe mechanism to coordinate the state of deployed components. This approach also allows to paralyze the deployment and facilitates fault detection. [9] is comparable to [4] which provides a very low level API to the deployer for its improvement. [25] presents Engage, a deployment framework which is very close to [26], it allows the user to only express a partial installation specification and it generates the full one. In contrast, our system provides a comparable feature since it is able to deploy a stack of software from a partial specification. [11] motivates the use of Model Driven Engineering as we do in our framework to build a useful multi-cloud platform. Therefore, it introduces CloudML which can be seen as a sub-part of our DSL. [13] focus on the migration of enterprise applications to hybrid cloud while considering enterprises constraints such as price, location, etc.

Very few solutions are close to our approach. Most of them are proprietaries and do not provide detailed documen-

\footnotetext{
${ }^{1}$ http://roboconf.net/
}

tation about their internal functioning. Cloudify [14] statically provides the possibility to use a number of cloud platforms. Unfortunately, it does not allow to deploy an application within different cloud platforms at the same time. Indeed, it is not able to exchange dependency information or components activation state across different clouds at runtime. Therefore, a deployment which requires participation of a private cloud (e.g. financial applications) is not possible with Cloudify. Regarding the description of applications and execution environment, Cloudify proposes a quite simple approach like other solutions. It does not consider the possible hierarchy of an application. RightScale [15] is another proprietary and commercial solution for deploying applications on the cloud. It proposes a set of applications templates (e.g. JEE) which can be improved by the deployer. Therefore, it does not allow the integration of new templates. No DSL is provided to the deployer as well. Scalr and EnStratus [16], [22] provide solutions in the same vein as RightScale.

In summary, all these solutions are not able to address the challenging we presented for many reasons. (1) They do not provide a hierarchical language which allows user to express the application in a stack way. They are limited to three levels of description: physical machine, VM and software. Therefore, a fine-grained installation mechanism is not allowed. (2) Most of these solutions are not improvable. They address either a well-known application or cloud platform. Few of them which are adaptable require a high expertise for the deployer though. In addition, they are not able to target at the same time more several cloud platforms, especially at runtime. Our framework exhibited in next part overcomes these two problems.

\section{A GENERIC MULTI-CLOUD DEPLOYMENT FRAMEWORK}

\section{A. Overview}

This part dictates our solution which is a deployment framework for multi-cloud, but not only. It allows to describe distributed applications and handle deployment automatically of the entire application or a part of it. The objective of this framework is to be improvable with a micro kernel which is the core of the whole system. This kernel implements all necessary mechanism to plug new behaviours for addressing new applications and new execution environment. Moreover, it supports scaling and dynamic (re)configuration natively. This provides a lot of flexibility and allows elastic deployments. The framework architecture is made up of several modules, which is simplified in Fig. 1 and explained more detail as follows. (1) The Deployment Manager (or DM) is an application in charge of managing VM and the agents (see below). It acts as an interface to the set of VMs or devices. It is also in charge of instantiating VMs in the IaaS and physical machines such as embedded boards; (2) The Agent is a software component that must be deployed on every VM and device on which we want to deploy or control something. Agents consume plug-ins to delegate the manipulation of Software instances. The plugins can be either life cycle management plug-ins that support disparate implementation languages or frameworks such as Bash, Puppet [23], Chef [24] OSGi [20], etc. or federated PaaS plug-ins such as Heroku [17] or CloudBees [18] drivers. The plug-ins can be flexibly plugged into the DM's kernel or agent. To not reinvent the wheel, it reuses existing and robust 


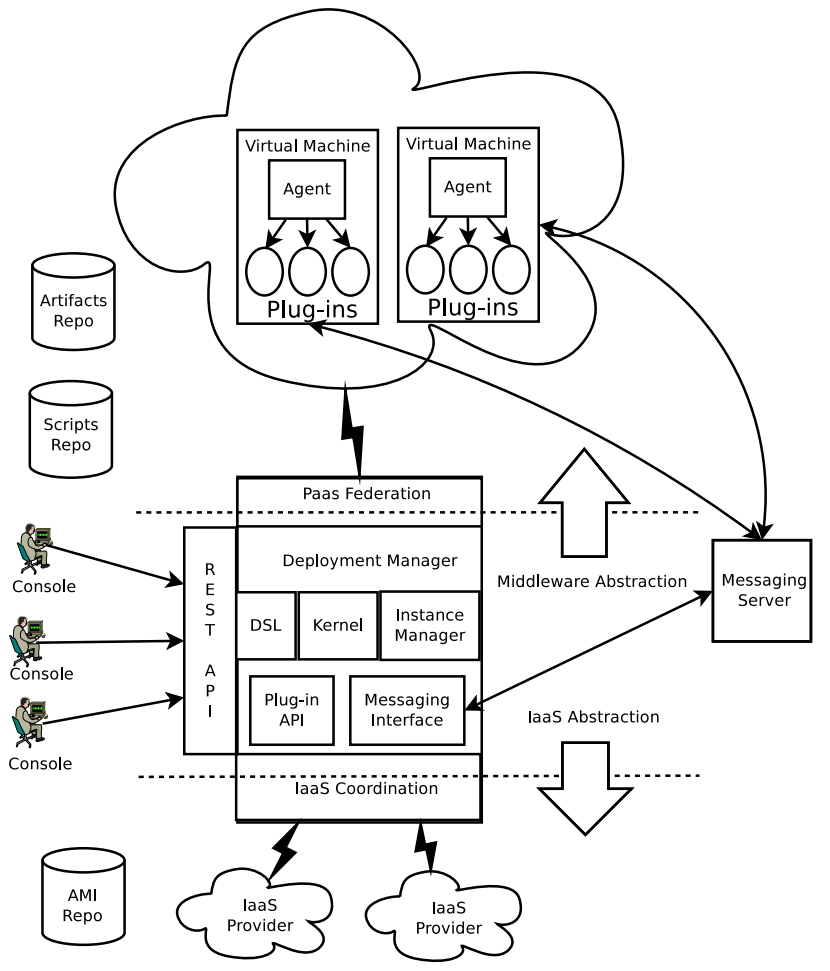

Fig. 1: Simplified architecture of the novel framework.

solutions such as Vagrant [28] or Docker [27]. The agents communicate with each other through an asynchronous messaging server; (3) The SoftwareInstanceManager is developed as a plug-in to generate software life-cycle management on different software platform and monitor software instances themselves; (4) The Messaging Server is the key component acting as distributed registry of import/export variables that enable communications between the DM and the agents. It includes the message definitions, the interface to interact with a given messaging server (currently RabbitMQ [19]) and their implementations. The DM and the agents always communicate asynchronously through this server; (5) The Artifact and VM Image repositories are responsible for distribution software packages (i.e. artifact) and VM's image, respectively. Artifact repositories can be managed locally or retrieved from public repositories such as Maven center or NPM. Image repository is a database to map each required VM image of each IaaS to corresponding infrastructure components. The required VM image can be an image available in the VM image marketplace provided by IaaS or a pre-built image created manually or automatically (e.g. using Docker or Vagrant); (6) Eventually, an admin console is required to control the DM. A shell-based console has been developed to interact with the DM through REST. It contains utilities to transform Java beans into JSON.

The framework is a distributed technology, based on AMQP [21] and REST/JSON. It is both IaaS and PaaSagnostic. Many well-known IaaS are advocated including OpenStack, Amazon Web Services, Microsoft Windows Azure, VMware vSphere, as well as a "local" deployment plug-in for on-premise hosts. In the PaaS aspect, not only potential type of applications are tensely brought up to the Cloud such as OSGi or Internet of Things (i.e. IoT) [29] but also state-ofthe-art PaaS are purposefully encompassed such as Heroku, Google App Engine and CloudBees. Its architecture allows to satisfy most of state-of-the-art requirements of a modern multicloud PaaS such as dynamic dependency resolution, concurrent deployment, genericity, multi-cloud distributed deployment, middleware-orientation, modularity, extensibility, portability, scalability and reusable/configurable deployment plan.

\section{B. A Hierarchical Architecture with DSL}

The aforementioned framework is designed to see a distributed application as a set of "components", and as a group of "instances" of these components. Let us take as an example the three-tier distributed application "Apache-Tomcat-MySQL". "Apache" is a component, while an install of Apache on a particular machine is an instance. Another install of Apache on another machine is another instance. Besides, our system is built to see distributed application as a group of components that each one exchanges a group of simple data between each other. Data can be either strings or more complex objects. Components of a distributed application are composed of variables as for example the IP address or the port used. Parts of those variables may be needed by other components of the application, they are named "exported vars", while vars coming from other components of the application are named "imported vars". Moreover, definition of a component can be inherited by definition of another according to object-oriented design. It inherits all import/export vars and default values. For instance, Tomcat component can inherit properties of a generic "Application Server" component.

Now that we have a far view of an application, let us explain more precisely what are its components. In the above example, Apache in this case simply imports variables coming from Tomcat: IP and port of the application server. As we said earlier, we define elements (component and instance) as having a set of exported and imported variables. In this case of Apache there are only imported variables. A sample of Apache component under the framework's language could be find in Fig. 2a left.

This portion is made up of several regions. alias: A component declaration. It is mandatory and provides a humanreadable name. installer: A component property. It is mandatory and designates the plug-in that will handle the life cycle of component instances. In this example, we are using puppet implementation. imports: lists the variables this components needs to be resolved before starting. Variable names are separated by commas. They are also prefixed by the component that exports them. As an example, if Tomcat exports the ip variable, then a depending component will import Tomcat.ip. On the other hand, MySQL does not import data from other components, it is the only one exporting data which are its IP and port. The definition of MySQL is found at in Fig. 2a right.

Here has a minor different from the exports which lists the variables this component makes visible to other components. The "ip" is a special variable name whose value will be set by the agents at runtime. All the other variables should specify a default value. In terms of model and configuration files, the framework has the following concepts: (1) The application descriptor contains meta-information of the application such as name, version qualifier and description; (2) The graph is in fact a set of connected components. It defines Software components which go from the (virtual) machine, cloud platform to 
(a)

\begin{tabular}{l|l}
\hline \# Apache Load Balancer & \# MySQL database \\
Apache \{ & MySQL \{ \\
alias: Apache Load Balancer; & alias: MySQL; \\
installer: puppet; & installer: bash; \\
imports: Tomcat.portAJP, & exports: ip, \\
Tomcat.ip; & port = 3306; \\
\hline
\end{tabular}

(b)

\begin{tabular}{|c|c|}
\hline $\begin{array}{l}\# \text { Tomcat app srv } \\
\text { Tomcat }\{ \\
\\
\text { alias: Tomcat; } \\
\text { installer: puppet; } \\
\text { exports: ip, } \\
\text { portAJP = } 8009 ; \\
\text { children: Rubis; }\end{array}$ & $\begin{array}{l}\text { \# RUBiS Application } \\
\text { Rubis \{ } \\
\text { alias: RUBiS war; } \\
\text { installer: java-servlet; } \\
\text { imports: MySQL.port, } \\
\text { MySQL.ip; } \\
\text { \} }\end{array}$ \\
\hline$\}$ & \\
\hline
\end{tabular}

Fig. 2: Examples of component of (a-left) Apache; (a-right) MySQL; (b-left) Tomcat; (b-right) Rubis.

the application package. The graph defines both containment and runtime relations. Two kinds of relations are defined as follows: (a) Containment means a component can be deployed over another one. As an example, a Tomcat server can be deployed over a VM. Or a web application (WAR) can be deployed over a Tomcat server (see Fig. 2b); (b) Runtime relations refer to components that work together. For instance, a web application needs a database. More specifically, it needs the IP address and the port of the database. Generally, this information is hard-coded whereas our system can instead resolve them at runtime and update components through the configuration or management APIs (e.g. JMX, REST). As an example, Apache, Tomcat and MySQL can be deployed simultaneously. Tomcat will be deployed but will not be able to start until it knows where is the database. Once the database is deployed and started, the system will update Tomcat configuration so that it knows where is MySQL. This is what dependency resolution at runtime makes possible; (3) If the graph defines relations between components, instances represent concrete components. Like a Java class, a component is only a definition. It needs to be instantiated to be used. Predefined instances aim at gaining some time when one wants to deploy application parts. As an example, the deployer could have defined a Tomcat component in the graph, and have two instances, one deployed on machine $\mathrm{A}$, and another on machine $B$ and other two on machine $C$. These would be four instances of the same component. The rules that apply to them are deduced from the graph, but they have their own configuration. An application is designed to see as hierarchy of components. The main motivation of hierarchy is to keep track of where instances are implemented in the system. It helps to make right decisions in dynamic deployment. A natural example of parent/children relationship of components of an OSGi application is depicted in Fig. 3.

There is a new important field: children which lists the components that can be instantiated and deployed over this component. In the example above, it means we can deploy Karaf over a VM instance. In turn, Joram and JNDI can be deployed over instances of Karaf.

While the hierarchical model resolves the containment relations (i.e. vertical relationship) among components at different layers and the export/import variables model is responsible for solving the runtime relations (i.e. horizontal relationship)

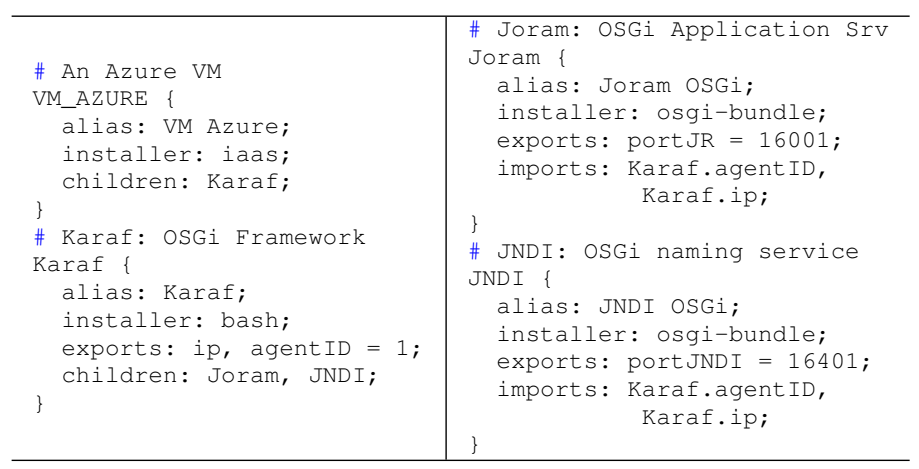

Fig. 3: Example of components of an OSGi application.

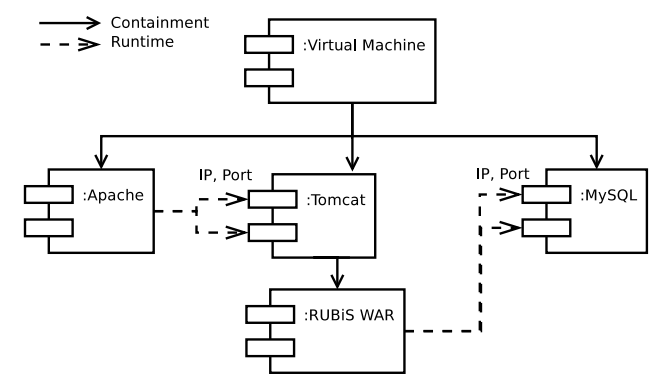

Fig. 4: Illustration of relationships among components.

amongst components at the same tier, a bi-color Graph put everything together using the DSL. What is modelled in the graph is really a user choice. Various granularity can be described. It can goes very deeply in the structure of application or bundle components together such as associating a given WAR with an application server. Multi-IaaS is supported by defining several root components. Each one will be associated with various properties (e.g. IaaS provider, VM type). In fact, the deployer can define a single graph, or a collection of graphs. The interest of having several graphs is to define virtual appliances.

\section{Deployment Process}

1) Initial Deployment Process: We use three-tier example to understand the way our framework works. As mentioned, dependencies between components is presented in Fig. 4. The goal is to deploy Apache, MySQL, Tomcat, Rubis instances on separate VMs that similar to Fig. 5b. The target software on Tomcat named RUBiS [36] which is a JEE application based on servlets, which implements an auction web site modelled on eBay. RUBiS defines interactions such as registering new users, browsing, buying or selling items. The updating the configuration files is performed as soon as dependencies can be resolved (e.g. when it is aware of the MySQL IP/port, this information will be sent to the Rubis nodes, so they can update its configuration and start). The application components (MySQL, Tomcat, Apache, Rubis) are defined as Fig. 5a. The VMs from disparate Cloud providers are supposed to support the deployment of either Apache, Tomcat, MySQL or Rubis components and each component is described in terms of imports/exports. With this description, the system knows when a deployed component can be started. It is when all its imports are resolved.

2) Redeployment Process: It often happens when everything is running, we need to create a new instance to adapt to changes from environment. It means the running system needs 
(a)

\begin{tabular}{|c|c|}
\hline $\begin{array}{l}\text { \# An Azure VM } \\
\text { VM_AzURE \{ } \\
\text { alias: VM on Azure; } \\
\text { installer: iaas; } \\
\text { children: Tomcat, } \\
\text { Apache, MySQL; } \\
\text { \} } \\
\text { \# An EC2 VM } \\
\text { VM_EC2 \{ } \\
\text { alias: VM on EC2; } \\
\text { installer: iaas; } \\
\text { children: Tomcat, } \\
\text { Apache, MySQL; } \\
\text { \} A VMware VM } \\
\text { \#M_VMWARE \{ } \\
\text { alias: VM on VMware; } \\
\text { installer: iaas; } \\
\text { children: Tomcat, } \\
\text { Apache, MySQL; } \\
\text { \} MySQL } \\
\text { MySQL \{ } \\
\text { alias: MySQL; } \\
\text { installer: puppet; }\end{array}$ & $\begin{array}{l}\text { exports: ip, port }=3306 ; \\
\text { \# Tomcat is container of Rubis } \\
\text { Tomcat \{ } \\
\text { alias: Tomcat; } \\
\text { installer: puppet; } \\
\text { exports: ip, } \\
\text { portAJP = } 8009 ; \\
\text { children: Rubis } \\
\text { \} } \\
\text { \# Apache Load Balancer } \\
\text { Apache \{ } \\
\text { alias: Apache Load Balancer; } \\
\text { installer: puppet; } \\
\text { imports: Tomcat.portAJP, } \\
\text { Tomcat.ip; } \\
\text { \} RuBis Application } \\
\text { Rubis \{ RUBis war; } \\
\text { alias: Rerlet; } \\
\text { installer: java-servlet; } \\
\text { imports: MySQL.port, } \\
\text { \} MySL.ip; } \\
\text { \} }\end{array}$ \\
\hline
\end{tabular}

(b)

\begin{tabular}{|c|c|}
\hline $\begin{array}{l}\text { \# A VM Azure with Apache } \\
\text { instanceof VM_AzURE \{ } \\
\text { name: vm-azure-apache; } \\
\text { instanceof Apache \{ } \\
\text { name: apache; } \\
\text { \} } \\
\text { \# A VM EC2 with Tomcat } \\
\text { instanceof VM_EC2 \{ } \\
\text { name: vm-ec2-tomcat-1; } \\
\text { instanceof Tomcat \{ } \\
\text { name: tomcat-1; } \\
\text { instanceof Rubis \{ } \\
\text { name: rubis-1; } \\
\text { \} }\end{array}$ & $\begin{array}{l}\text { \# A VM VMware with Tomcat } \\
\text { instanceof VM_VMWARE \{ } \\
\text { name: vm-vmware-tomcat-2; } \\
\text { instanceof Tomcat \{ } \\
\text { name: tomcat-2; } \\
\text { instanceof Rubis \{ } \\
\text { name: rubis-2; } \\
\text { \} } \\
\text { \} } \\
\text { \# A VM VMware with MySQL } \\
\text { instanceof VM_VMWARE \{ } \\
\text { name: vm-vmware-mysql; } \\
\text { instanceof MySQL \{ } \\
\text { name: mysql; } \\
\text { \} }\end{array}$ \\
\hline
\end{tabular}

Fig. 5: (a) Example of a DSL Graph of components; (b) Example of a DSL Graph of instances.

to indicate the software component to instantiate, devise a name and define where it should go. In this particular example, we create a new Tomcat instance. Given our configuration files, it can merely go under a VM one. We can either reuse an existing instance or create another VM instance. In this scenario, we will take the second option. We only add instances in the model. They are not started, and not even deployed. We ask the DM to deploy and start all of them. First, the DM creates the new VM. Once it is up, the DM sends the deployment command to the agent inside this VM and a new Tomcat instance is deployed over the virtual machine. The agents then publishes the exports (i.e. a new Tomcat instance with a port and IP address). Since the Apache load balancer imports such components, it is notified a new Tomcat arrived. The agent associated with the Apache VM invokes a plug-in to update the configuration files of the Apache server. Therefore, the load balancer is now aware of two Tomcat servers. If configured in round-robin, it will invoke alternatively every Tomcat server when it receives a request. It is worth noting that real magic here is the asynchronous exchange of dependencies between software instances whereas the deployment and life cycle actions are delegated to plug-ins.

\section{EXPERIMENTAL EVALUATION}

To validate the soundness of the architecture, we conducted a number of experiments with scenarios selected from practical use cases.

\section{A. Experiment 1}

The first type of experiments demonstrates the advantage of hierarchical structure which is aforementioned. For this experiment, EC2 was the target cloud.

1) Scenario and Requirements: We performed this experiment with an OSGi application. Regularly, an OSGi application consists of one or several OSGi containers (e.g. Karaf, Felix, Equinox) providing runtime environment and management platform for OSGi bundles such as Joram, JNDI. We used two instances of EC2 m3.medium, each hosts 2 instances of Karaf. Each Karaf inside a VM is customized to choose either Felix or Equinox as underlying OSGi framework and hosts an instance of Joram (an OSGi JMS-supported server), or an instance OSGi JNDI or a JMS OSGi client (publisher/subscriber). Deployment of Joram, JNDI and OSGi JMS clients is handled by the "osgi-bundle" installer, specific to this type of application. We chose Cloudify as comparative objective because it also offers scripting language that can be used to express the structure of a distributed application. Our platform sees hierarchy of this application as depicted in Fig. 6 top, whereas Fig. 6 bottom shows flat structure of the same application in Cloudify.

2) Results: With its hierarchical DSL and its extensibility, users only have to write one deployment plan for EC2, one for Karaf OSGi container and reuse one plan for multiple OSGI bundles (Joram, JNDI, subscriber, publisher). With Cloudify, 6 deployment plans are needed, each one for each component (EC2, Karaf, Joram, JNDI, Subscriber, Publisher). Thus Cloudify users have to write twice more deployment plans.

\section{B. Experiment 2}

The second type of experiments gives some evidence for the correctness of the multi-cloud deployment feature. We compare deployment time of a Storm [30] cluster (an Event Stream Processing (ESP) application) on a multi-cloud platforms using our framework on the one hand and a manual configuration following installation guide from original owner on the other hand. Storm is a part of a global solution for Ubilitycs [31] (ubiquitous big data analytics for IoT). Storm consists of Zookeeper cluster, Nimbus server, Storm supervisors and requires installation of JZMQ, ZeroMQ and Python. The experiment was conducted in a multi-cloud environment combining two public clouds (EC2 and Azure) and a private Cloud (VMware vSphere). Three IaaS plug-ins for these clouds have been developed to provide coordination among the three IaaS providers. Each plug-in implementation always implements an interface of the framework's plug-in API. The LOCs (lines-of-code) for the EC2 plug-in is 202, 393 for Azure, and 157 for VMware vSphere. Zookeeper cluster was installed on EC2, Nimbus server on Azure and Storm supervisors on our VMware vSphere data-center to take advantage of our computing strength. In this experiment, the time for installing Storm manually is compared with the time to automate its installation using our solution. The online installation guide of Storm is 8 pages long containing many external links to resource document of relevant dependencies. One of the 
authors who had no knowledge about and attempted to install Storm previously tried to do manual installations. It took him about 6 hours the first time, 3 hours and 30 minutes the second time, and up to 1 hour from the third one. Actions eating effort time were reading imprecise instructions, resolving environment issues, seeking/downloading the required dependencies and debugging problems.On the novel framework side, time mainly devotes for writing deployment plan of Zookeeper, Nimbus, Supervisors, JZMQ, ZeroMQ and Python. About 120 LOC have been written for scripts of all Storm's components. After installation, Storm can be managed (deploy, start, stop, undeploy, update) by our platform and automatically connect to other applications. The total development time for automation of Storm was about 2 hours 55 minutes. This time was divided into 30 minutes for component type's design, 70 minutes for writing scripts and 75 minutes for debugging and testing. If the required packages are downloaded from the Internet, install of Storm needs 20 minutes and around 7 minutes if the packages are retrieved from a local repository. The automation of the Storm installation empowers Storm developers deploy their existing applications on multi-cloud with slight changes and no need to understand details of the middleware. It warrants a repeatable procedure and can be used as a part of larger deployments (e.g. Ubilytics).

\section{CONCLUSION}

The novel distributed framework we propose in this paper is a generic (administrate any kind of application), extensible, multi-cloud, scalable, and fine-grained reconfigurable deployment framework. It is based on a lightweight kernel which implements basic administration mechanisms. Its simplicity (regarding its implementation) combined with its component based approach ease its improvement by any deployer. More important, it provides a hierarchical DSL (Domain Specific Language) for a fine-grained expression of applications and execution environments. This allows it to achieve fine-grained level of administration (physical machine, VM, software within VM, other stack inside software). Our experiments about OSGi, Storm cluster validate all the framework's features on hybrid cloud. In addition, it outperforms most popular deployment frameworks offering DSL like Cloudify in terms of reusability. A further enhancement would be the implementation of a way to facilitate the integration of more sophisticated reconfiguration policies (e.g. for scalability, fault tolerance).

\section{ACKNOWLEDGMENT}

The work reported in this paper benefited from the advocate of the French National Research Agency through projects CtrlGreen (ANR-11-INFR-0012).

\section{REFERENCES}

[1] Jeffrey O. Kephart and David M. Chess, 'The vision of autonomic computing," Computer 36(1) 2003.

[2] Jeffrey O. Kephart, 'Autonomic Computing: The First Decade," keynote ICAC 2011

[3] Kyle Oppenheim and Patrick McCormick, 'Deployme: Tellme's Package Management and Deployment System,” LISA 2000.

[4] Alain Tchana, Suzy Temate, et al. 'TUNeEngine: An Adaptable Autonomic Administration System," SCSE 2013.

[5] Dearle A., 'Software Deployment, Past, Present and Future," FOSE 2007.

[6] Hyun Jung La and Soo Dong Kim, 'Dynamic Architecture for Autonomously Managing Service-Based Applications," SCC 2012.

[7] Eric Yuan, Naeem Esfahani, and Sam Malek, 'A Systematic Survey of Self-Protecting Software Systems,” TAAS 2014.

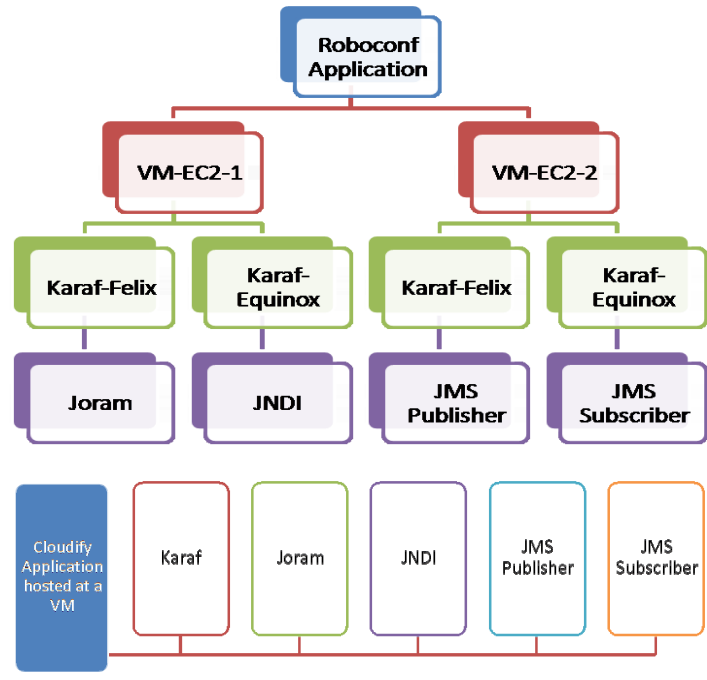

Fig. 6: OSGi use case: Novel framework's hierarchical view vs. Cloudify flat view.

[8] Flexiscale, 'http://www.flexiscale.com," visited on August 2014.

[9] Fawaz Paraiso, Nicolas Haderer, et al. 'Federated Multi-Cloud PaaS Infrastructure," CLOUD 2012.

[10] Deepal Jayasinghe, et al. 'AESON: A Model-Driven and Fault Tolerant Composite Deployment Runtime for IaaS Clouds," SCC 2013.

[11] Nicolas Ferry, et al. 'Towards model-driven provisioning, deployment, monitoring, and adaptation of multi-cloud systems," CLOUD 2013.

[12] Tao Zou, Ronan Le Bras et al. "ClouDiA: A Deployment Advisor for Public Clouds," VLDB Endowmen 6(2) 2012.

[13] Mohammad Hajjat, et al. 'Cloudward Bound: Planning for Beneficial Migration of Enterprise Applications to the Cloud," SIGCOMM 2010.

[14] 'http://www.cloudifysource.org," visited on August 2014

[15] RightScale, 'www.rightscale.com," visited on August 2014.

[16] Scalr, "http://www.scalr.com," visited on August 2014.

[17] Heroku, "https://www.heroku.com/," visited on August 2014.

[18] CloudBees, 'http://www.cloudbees.com/," visited on August 2014.

[19] RabbitMQ, "https://www.rabbitmq.com/," visited on August 2014.

[20] OSGI, 'http://www.osgi.org/Main/HomePage," visited on August 2014.

[21] AMQP, 'http://www.amqp.org/," visited on August 2014.

[22] EnStratus, "http://www.enstratius.com," visited on August 2014.

[23] Puppet, "http://puppetlabs.com," visited on August 2014.

[24] Chef, "http://www.getchef.com/chef," visited on August 2014.

[25] Jeffrey Fischer, Rupak Majumdar, and Shahram Esmaeilsabzali, 'Engage: a deployment management system," PLDI 2012.

[26] Broto L., Hagimont D., Stolf P., De Palma N., and Temate S., 'Autonomic management policy specification in Tune," SAC 2008.

[27] Docker, "https://www.docker.io," visited on August 2014.

[28] Vagrant, "http://docs.vagrantup.com/v2/multi-machine/index.html," visited on August 2014.

[29] Jayavardhana Gubbi, et al. 'Internet of Things (IoT): A vision, architectural elements, and future directions," 29(7) FGCS 2013.

[30] Storm, 'http://storm-project.net," visited on August 2014

[31] Niklas Elmqvist, Pourang Irani, 'Ubiquitous Analytics: Interacting with Big Data Anywhere, Anytime," vol.46, no.4, pp.86,89, Computer 2013.

[32] Linagora, "http://www.linagora.com," visited on August 2014.

[33] Amazon EC2, "http://aws.amazon.com/ec2," visited on August 2014.

[34] Microsoft Azure, "http://windowsazure.com," visited on August 2014.

[35] OpenHAB, 'http://www.openhab.org," visited on August 2014.

[36] RUBiS, ‘http://rubis.ow2.org," visited on August 2014. 